\title{
Measuring Transition Readiness in Adolescents and Young Adults with Sickle Cell Disease Using the Transition Readiness Assessment Questionnaire
}

\author{
Anjali Oberoi ${ }^{1}$, Alyssa Patterson ${ }^{2}$, and Amy Sobota ${ }^{3}$ \\ ${ }^{1}$ Tufts University School of Medicine \\ ${ }^{2}$ Boston University School of Public Health \\ ${ }^{3}$ Boston Medical Center
}

May 19, 2021

\begin{abstract}
Background/Objectives: Adolescents and young adults (AYA) with sickle cell disease (SCD) face challenges related to the disease and its treatment. The Transition Readiness Assessment Questionnaire (TRAQ) is a self-report tool for assessing transition readiness for youth with special health care needs (YSHCN), including SCD. This study uses the TRAQ to understand transition readiness in patients with SCD treated at the Boston Medical Center, evaluates associations between TRAQ scores and transition outcomes (e.g., EDr, EDu), and compares TRAQ scores in this population with other YSHCN. Methods: We reviewed electronic medical records of AYA with SCD who completed the TRAQ in the pediatric hematology clinic between January 1, 2019, and March 1, 2020, and categorized healthcare encounters to calculate EDu and EDr. We used t-tests and ANOVA models to analyze mean TRAQ scores, sex, age, genotype, EDu, and EDr. Results: The sample was 45 AYA patients with SCD between 13 and 22 years old. The mean TRAQ score for the overall patient sample was 3.67. Mean TRAQ scores did not significantly vary by sex or genotype but did significantly increase with age. TRAQ scores were lower in the SCD population than in other YSHCN. TRAQ scores did not correlate to EDu or EDr. Conclusions: AYA patients with SCD have lower transition readiness than other populations of YSHCN. The age of 18 may not be the most reliable attribute of readiness, though older patients do have higher readiness. The relationship between TRAQ scores, EDr, and EDu is not clear and requires further evaluation.
\end{abstract}

\section{INTRODUCTION}

Sickle cell disease (SCD) is a group of genetic hematologic disorders with approximately 100,000 affected individuals in the United States. ${ }^{1}$ People with SCD experience symptoms and complications related to vasoocclusive episodes beginning in infancy, including stroke and increased risk of bacterial respiratory infections with subsequent acute chest syndrome. ${ }^{2,3}$ Fortunately, improved quality of care for children with SCD has reduced mortality and increased lifespan in recent years. Almost all children with SCD now reach adulthood in high resource countries. ${ }^{4}$ However, there is inadequate health system support for adolescents transitioning from pediatric to adult models of care.

Transition to adulthood is a time of extended exploration for all adolescents, and decisions made during this period influence adult functioning. ${ }^{5}$ Transition is a process aimed at preparing adolescents and young adults (AYA) to live independently. ${ }^{6}$ Transition includes transfer of care to an adult provider and further encompasses aspects of everyday adult living beyond the change of provider. ${ }^{7}$ AYAs with chronic diseases often consider health care a low priority compared to the other dynamic components of their transition to adulthood (e.g., education, employment, housing, and relationships) ${ }^{8,9}$ For patients with SCD, transition from pediatric to adult care should "maximize lifelong functioning and potential through the provision of 
high quality, developmentally appropriate health care services that continue uninterrupted as the individual moves from adolescence to adulthood." 10

Additionally, AYA with SCD present with unique health concerns related to the disease and its treatment. The frequency red blood cell transfusions and the proportion of patients receiving accompanying ironchelation therapy declines substantially during the transition period and remains low through adulthood. ${ }^{11}$ In contrast, the incidence of SCD-related complications increases steadily throughout the transition period and continues to rise throughout adulthood. Health care resource utilization for acute care needs and rehospitalization rates are highest for adolescent patients with SCD. ${ }^{12}$ Emergency Department utilization (EDu) and Emergency Department reliance (EDr) increase in patients with SCD during transitions to adult care, and remain high after the transition period throughout adulthood. ${ }^{13-15}$ EDu identifies frequent ED users due to increased need for care, while EDr identifies the proportion of ED visits in relation to all ambulatory visits, distinguishing those who might not have adequate access to outpatient care. ${ }^{15}$ The relationship between increased EDr by AYA patients with SCD and clinical factors (e.g., genotype, comorbidities), financial and socioeconomic status, and psychosocial factors is documented; however, the relationship between EDu and EDr and transition readiness has not been explored. ${ }^{16-18}$

The Transition Readiness Assessment Questionnaire (TRAQ) is a skill-focused, self-report tool for assessing the developmental stage of transition readiness for youth with chronic medical conditions, including SCD. The TRAQ has been validated in Youth with Special Healthcare Needs (YSCHN) and evaluated in other disease groups, including patients with type 1 diabetes and inflammatory bowel disease; however there is limited research in patients with SCD. ${ }^{19-21}$ AYA with SCD often lack the disease knowledge necessary to transition care, and would benefit from an assessment for transition readiness. ${ }^{22}$

Our primary objective in this study was to assess transition readiness in patients with SCD treated at the Boston Medical Center (BMC) using the TRAQ. Secondary objectives included evaluating for associations between TRAQ scores and transition outcomes such as EDr and EDu and comparing TRAQ scores in this population with the larger population of YSHCN. We hypothesize that there will be a correlation between low TRAQ scores and high EDu and EDr. These results inform preparation for and timing of transfer to adult care and allow for assessment of the impact of strategies to improve readiness on clinically relevant and easily measurable outcomes (e.g., EDr, EDu).

\section{METHODS}

\section{Overview}

We performed a single-center, retrospective study of AYA patients with SCD, to assess transition readiness using the TRAQ. Inclusion criteria were patients with SCD ages 13-22 years old receiving care in the pediatric hematology clinic at BMC. Exclusion criteria were non-English speaking and the inability to complete the TRAQ due to cognitive impairment. The Boston University Medical Center Institutional Review Board granted an exemption for this study.

\section{Data Collection}

We reviewed electronic medical records (EMR) of all AYA with SCD who completed the TRAQ in the pediatric hematology clinic between January 1, 2019, and March 1, 2020. We recorded demographic data and TRAQ scores. Healthcare encounters from the 12 months preceding completion of the TRAQ were recorded from the EMR and categorized.

\section{TRAQ Completion}

Patients completed the validated TRAQ as part of their medical visit to the pediatric hematology clinic and discussed it with their physician. The pediatric hematology clinic serves approximately 180 patients with SCD from birth through 22 years of age. Between January 1, 2019 - March 1, 2020, 45 out of 97 eligible of patients ages 13-22 completed the TRAQ.

\section{Independent Variables}


The TRAQ contains 20 self-report questions that measure transition readiness using a 5-point Likert scale. The questionnaire includes five domains: Appointment Keeping, Tracking Health Issues, Managing Medications, Talking With Providers, and Managing Daily Activities. ${ }^{19}$ Demographic data for each patient included age in years, sex, and SCD genotype.

\section{Dependent Variables}

All in-person healthcare encounters at BMC from the 12 months preceding completion of the TRAQ were recorded and categorized as outpatient or Emergency Department (ED). We recorded ambulatory primary care, hematology, and other subspeciality (cardiology, pulmonary, etc.) appointments as outpatient visits. We only recorded visits that the patient attended (i.e., we did not record the number of missed appointments, "no shows" or telephone encounters). We calculated EDr as the percentage of healthcare encounters in the ED (the number of ED visits divided by the number of ED and outpatient encounters). ${ }^{15}$ We categorized high ED utilization as over three ED presentations per year and defined high EDr as $>0.33$ (i.e., more than one-third of health care received in the ED). ${ }^{11,23}$

\section{Statistical Analysis}

Continuous demographic variables were defined using mean and standard deviations, whereas categorical demographic variables were given as percentages. Unpaired t-tests and one-way ANOVA models were performed to compare mean TRAQ scores by sex, age, genotype, EDu and EDr. Similar unpaired t-test and one-way ANOVA models were used to compare mean EDu by sex, age, and genotype and mean EDr by sex, age, and genotype. Finally, multiple linear regression was used to determine the association between overall TRAQ score and high EDu controlling for age, sex, and genotype. The significance level was set at 0.05 for all analyses.

We also compared mean TRAQ scores among our study population of adolescent patients with SCD at BMC against other study populations from the literature, including adolescents with Type 1 Diabetes (T1DM), adolescents and young adults with inflammatory bowel diseases (IBD), YSHCN, and healthy youth (Fig 1).

\section{RESULTS}

\section{Patient Demographics}

The study sample was 45 AYA patients with SCD between 13 and 22 years old. Overall, the mean patient age was 17.1 years, $53.3 \%$ were female, and $71.1 \%$ have HbSS genotype (Table 1). The mean TRAQ score was 3.67, the mean EDu was 1.2 visits (Table 1), and EDr (proportion of ambulatory healthcare visits took place in the ED) was $10.6 \%$ of (Table 2 ).

\section{Mean TRAQ Scores (Table 2)}

The mean TRAQ score for the overall patient sample was 3.67 (Table 2). The mean TRAQ scores were higher among older age groups, female patients, and those with the HbSS genotype. Unpaired t-test and ANOVA analyses did not find the difference in mean TRAQ score by sex, genotype, EDu, or EDr statistically significant. However, they did find statistical significance $(\mathrm{p}=0.0004)$ in the mean TRAQ score by age.

We also examined mean TRAQ scores by individual domains: Managing Medications, Appointment Keeping, Tracking Health issues, Talking with Providers, and Managing Daily Activities. Mean domain scores were highest among the 20-22 age group for most categories, except Talking with Providers where, where patients ages 16-19 had the highest score (Fig 2).

We compared mean TRAQ scores among our study population of adolescent patients with SCD at BMC against other study populations from the literature. This comparison showed that among these patients with SCD treated at BMC, youth with Type 1 Diabetes, youth with IBD, YSCHN overall, and healthy youth, healthy youth had the highest average TRAQ scores (mean =3.93). Healthy youth lead in the component scores of Appointments Keeping $($ mean $=4.07)$ and Managing Daily Activities (mean =4.63). Patients with IBD had the highest TRAQ component score in Talking with Providers (mean $=4.86$ ) and Tracking Health 
Issues (mean $=3.56)$ (Table 7). YSHCN had the highest TRAQ component score in Managing Medications (mean $=3.97$ ). Our study population of AYA with SCD did not have the average highest score in any of the component categories; specifically, they had the lowest TRAQ scores in Appointment Keeping (mean = 2.91) and Tracking Health Issues (mean = 2.96) (Fig 1).

\section{Mean EDr and EDu (Table 3)}

The mean EDr was highest for those patients in the 16-19 years of age group, among female patients and those with the HbSS genotype. EDu was highest among the 16-19 age group compared to the other age groups. EDu was higher among male patients versus the average for females. Mean EDu was also highest among those with the HbSS genotype compared to the other genotypes. Unpaired t-test and ANOVA analyses did not find the difference in mean EDr among age, sex, or genotype statistically significant, nor did they find the difference in mean EDu among these demographics statistically significant.

\section{Multivariate Analysis (Table 4)}

A multiple linear regression controlling for demographic data found that, on average, the overall TRAQ score increases by 0.24 points for every one-year increase in age. There was no significant evidence of an association between sex, genotype, or EDu and TRAQ score, adjusting for these variables and age.

\section{DISCUSSION}

This is one of the first studies to report on transition readiness specifically in AYA with SCD using the TRAQ. A TRAQ score of 4 is used clinically an indicator of readiness to transition to adult care; the mean TRAQ score in our sample was 3.67, below the target level, and emphasizes the need for increased efforts regarding transition planning and care for adolescents and young adults with SCD.

This study indicates that age is a useful demographic facture to understand and measure AYA with SCD's transition readiness. Transfer to adult care is usually at the age of 18 , however, the present findings indicate that many patients did not achieve readiness to transition, supporting the use of age alone should not be the criteria for leaving pediatric care. Only the oldest patients (age 20-22) in our population met the transition readiness benchmark, both in terms of mean overall TRAQ score and within each domain. Younger patients (ages 16-19) achieved a mean score above 4 in the Talking with Providers and Managing Daily Activities domains, while the youngest subgroup (age 13-15) achieved mastery only in Managing Daily Activities. These findings are consistent with previous research that older patients score higher on the TRAQ as some self-management skills improve with age. ${ }^{19}$

Examination of TRAQ scores by item also allows for identifying strengths and weaknesses regarding readiness skill acquisition. AYA reported the lowest mastery levels areas in Appointment Keeping and Tracking Health Issues. These transition readiness areas represent modifiable behaviors that can be addressed with patients and families within the context of clinical care, including education interventions to enhance self-management skills with these specific goals in mind. Process measures prior to transition, such as having a written, selfdirected transition plan and portable, patient-centered medical record, have previously been identified as important components of quality care in SCD, and could be implemented in the setting of transition. ${ }^{24}$

In our study, patients scored lower on transition readiness measures than other YSHCN in every domain, lower than patients with IBD in every domain, and lower than patients with T1DM in every domain except for Appointment Keeping and Tracking Health Issues. There are several possible explanations for our patients' lower scores. Difficulties related to adverse social determinants of health likely impede the ability to adhere to SCD treatment plans as adolescent transitions to adulthood by limiting the degree to which adolescents can focus on SCD in the setting of other challenges. ${ }^{25,26}$ Furthermore, in the US, a majority of SCD patients are Black or another minority. ${ }^{27}$ Disability-related to SCD, enhanced by health crises at the time of transition, aggravate existing structures of racism. Interventions aimed at helping youth keep their appointments such as peer transition navigators, or larger reliance on Telehealth, may be helpful in this population.

A strength of this study is that it is the first in our knowledge to correlate TRAQ scores and clinical data 
in patients with SCD. This paper examines the association of EDu and EDr and transition readiness scores assessing self-management and self-advocacy skills. EDu and EDr are readily available measures that identify increased need for ED services and lack of access to quality chronic disease care at the time of transition. ${ }^{15}$ The associations between EDu and EDr and transition readiness were insignificant, contradicting the hypothesis that there would be a correlation between low TRAQ scores and high EDu and EDr. ${ }^{28}$ However, this study is limited by a small sample size, with the restricted, convenient sample of patients who came for visits at BMC. Selection bias could have been present as only patients who came for routine assessment were part of the study. The increase in EDu and EDr in AYA patients with SCD is likely multifactorial.

In conclusion, patients with SCD have lower transition readiness than other populations. The age of 18 may not be the best indicator of readiness; however, older patients do have higher readiness and may benefit from further support at a critical age. Further evidence is needed to understand the relationship between transition readiness and health outcomes. Future studies should administer the TRAQ to a larger number of AYA patients with SCD. Longitudinal tracking of TRAQ scores and clinical data, such as EDr, over time may give greater insight into true impacts. Future work is also needed assess the association of TRAQ scores for AYA patients with SCD on other measures health and transition outcomes such as access to adult provers and completion of goals (e.g., education, jobs) in young adulthood.

\section{Conflict of Interest}

The authors have no conflicts of interest to disclose

\section{Acknowledgements}

AO was funded by the Fredrick and Bernadette Stohlman Student Research Award, Tufts University School of Medicine

AS received funding from the National Heart Lung and Blood Institute 1K23HL135436-01

\section{Data Availability Statement}

The data that support the findings of this study are available on request from the corresponding author. The data are not publicly available due to privacy or ethical restrictions.

\section{REFERENCES}

1. Hassell KL. Population Estimates of Sickle Cell Disease in the U.S.Am J Prev Med . 2010;38(4 SUPPL.). doi:10.1016/j.amepre.2009.12.022

2. Platt OS, Thorington BD, Brambilla DJ, et al. Pain in sickle cell disease: Rates and risk factors. $N$ Engl J Med . 1991;325(1):11-16. doi:10.1056/NEJM199107043250103

3. Caboot JB, Allen JL. Pulmonary complications of sickle cell disease in children. Curr Opin Pediatr . 2008;20(3):279-287. doi:10.1097/MOP.0b013e3282ff62c4

4. Quinn CT, Rogers ZR, McCavit TL, Buchanan GR. Improved survival of children and adolescents with sickle cell disease. Blood . 2010. doi:10.1182/blood-2009-07-233700

5. Osgood DW, Foster EM, Flanagan C, Ruth GR. On Your Own without a Net: The Transition to Adulthood for Vulnerable Populations. The John D. and Catherine T. MacArthur Foundation Series on Mental Health and Development .; 2005.

6. Blum RW. Transition to adult health care: Setting the stage. In:Journal of Adolescent Health . ; 1995. doi:10.1016/1054-139X(95)00073-2

7. Bryant R, Porter JS, Sobota A. APHON/ASPHO Policy Statement for the Transition of Patients With Sickle Cell Disease From Pediatric to Adult Health Care. J Pediatr Oncol Nurs . 2015;32(6):355-359. doi: $10.1177 / 1043454215591954$ 
8. Junge N, Migal K, Goldschmidt I, Baumann U. Transition after pediatric liver transplantation - Perceptions of adults, adolescents and parents. World J Gastroenterol . 2017;23(13):2365-2375. doi:10.3748/wjg.v23.i13.2365

9. Pyatak EA, Sequeira PA, Whittemore R, Vigen CP, Peters AL, Weigensberg MJ. Challenges contributing to disrupted transition from paediatric to adult diabetes care in young adults with Type 1 diabetes.Diabet Med . 2014;31(12):1615-1624. doi:10.1111/dme.12485

10. American Academy of Pediatrics American Academy of Family Physicians 83 American College of Physicians- American Society of Internal Medicine. A Consensus Statement on Health Care Transitions for Young Adults . Vol 110.; 2002. doi:10.1542/peds.110.6.S1.1304

11. Blinder MA, Vekeman F, Sasane M, Trahey A, Paley C, Duh MS. Age-related treatment patterns in sickle cell disease patients and the associated sickle cell complications and healthcare costs. Pediatr Blood Cancer . 2013;60(5):828-835. doi:10.1002/pbc.24459

12. Brousseau DC, Owens PL, Mosso AL, Panepinto JA, Steiner CA. Acute care utilization and rehospitalizations for sickle cell disease. JAMA - J Am Med Assoc . 2010;303(13):1288-1294. doi:10.1001/jama.2010.378

13. Blinder MA, Duh MS, Sasane M, Trahey A, Paley C, Vekeman F. Age-Related Emergency Department Reliance in Patients with Sickle Cell Disease. J Emerg Med . 2015;49(4):513-522.e1. doi:10.1016/j.jemermed.2014.12.080

14. Singh A, Yan K, Brandow AM, Panepinto JA. Longitudinal Trend in Emergency Department Reliance for Pain Among Sickle Cell Disease Patients in Wisconsin. J Pediatr Hematol Oncol . 2019;41(7):E438-E442. doi:10.1097/MPH.0000000000001533

15. Kroner EL, Hoffmann RG, Brousseau DC. Emergency Department Reliance: A Discriminatory Measure of Frequent Emergency Department Users.Pediatrics . 2010;125(1):133 LP - 138. doi:10.1542/peds.2009-0960

16. Glassberg JA, Wang J, Cohen R, Richardson LD, DeBaun MR. Risk Factors for Increased ED Utilization in a Multinational Cohort of Children With Sickle Cell Disease. Acad Emerg Med . 2012;19(6):664-672. doi:10.1111/j.1553-2712.2012.01364.x

17. Logan DE, Radcliffe J, Smith-Whitley K. Parent factors and adolescent sickle cell disease: associations with patterns of health service use. J Pediatr Psychol . 2002;27(5):475-484. doi:10.1093/jpepsy/27.5.475

18. Cronin RM, Hankins JS, Byrd J, et al. Risk factors for hospitalizations and readmissions among individuals with sickle cell disease: results of a U.S. survey study. Hematology . 2019;24(1):189-198. doi:10.1080/16078454.2018.1549801

19. Sawicki GS, Lukens-Bull K, Yin X, et al. Measuring the transition readiness of youth with special healthcare needs: Validation of the TRAQ - Transition readiness assessment questionnaire. J Pediatr Psychol . 2011;36(2):160-171. doi:10.1093/jpepsy/jsp128

20. Baldassano R, Ferry G, Griffiths A, Mack D, Markowitz J, Winter H. Transition of the patient with inflammatory bowel disease from pediatric to adult care: Recommendations of the North American society for pediatric gastroenterology, hepatology and nutrition. J Pediatr Gastroenterol Nutr . 2002;34(3):245-248. doi:10.1097/00005176-200203000-00001

21. Sawicki GS, Garvey KC, Toomey SL, et al. Preparation for transition to adult care among medicaidinsured adolescents. Pediatrics . 2017;140(1). doi:10.1542/peds.2016-2768

22. Speller-Brown B, Varty M, Thaniel L, Jacobs MB. Assessing Disease Knowledge and Self-Management in Youth With Sickle Cell Disease Prior to Transition. J Pediatr Oncol Nurs . 2019;36(2):143-149. doi: $10.1177 / 1043454218819447$

23. Aisiku IP, Smith WR, McClish DK, et al. Comparisons of High Versus Low Emergency Department Utilizers in Sickle Cell Disease. Ann Emerg Med . 2009;53(5):587-593. 
doi:https://doi.org/10.1016/j.annemergmed.2008.07.050

24. Wang CJ, Kavanagh PL, Little AA, Holliman JB, Sprinz PG. Quality-of-care indicators for children with sickle cell disease.Pediatrics . 2011. doi:10.1542/peds.2010-1791

25. Henize AW, Beck AF, Klein MD, Adams M, Kahn RS. A road map to address the social determinants of health through community collaboration. Pediatrics . 2015. doi:10.1542/peds.2015-0549

26. Walsh KE, Cutrona SL, Kavanagh PL, et al. Medication adherence among pediatric patients with sickle cell disease: A systematic review.Pediatrics . 2014. doi:10.1542/peds.2014-0177

27. Ojodu J, Hulihan MM, Pope SN, Grant AM. Incidence of sickle cell trait - United states, 2010. Morb Mortal Wkly Rep . 2014;63(49):1155-1158.

28. Brousseau DC, Gorelick MH, Hoffmann RG, Flores G, Nattinger AB. Primary Care Quality and Subsequent Emergency Department Utilization for Children in Wisconsin Medicaid. Acad Pediatr . 2009;9(1):3339. doi:10.1016/j.acap.2008.11.004

\section{FIGURE LEGENDS}

FIGURE 1 Mean TRAQ scores by domain compared with other YSHCN. AYA with SCD did not have the average highest score in any of the component categories. AYA with SCD had the lowest TRAQ scores in Appointment Keeping and Tracking Health Issues. Healthy youth had the highest overall TRAQ scores, and highest component scores of Appointments Keeping and Managing Daily Activities. Patients with IBD had the highest TRAQ component score in Talking with Providers and Tracking Health Issues. YSHCN had the highest TRAQ component score in Managing Medications.

FIGURE 2 Mean TRAQ scores by age group. Mean TRAQ scores were highest in the 20-22 age group for the Managing Medications, Appointment Keeping, Tracking Health issues, and Managing Daily Activities domains. Mean TRAQ scores for Talking with Providers were highest in the 16-19 age group.

\section{Hosted file}

MEASURING TRANSITION READINESS TRAQ Table 1 05.16.2021.pdf available at https://authorea.com/ users/414500/articles/522530-measuring-transition-readiness-in-adolescents-and-youngadults-with-sickle-cell-disease-using-the-transition-readiness-assessment-questionnaire

\section{Hosted file}

MEASURING TRANSITION READINESS TRAQ Table 2 05.16.2021.pdf available at https://authorea.com/ users/414500/articles/522530-measuring-transition-readiness-in-adolescents-and-youngadults-with-sickle-cell-disease-using-the-transition-readiness-assessment-questionnaire

\section{Hosted file}

MEASURING TRANSITION READINESS TRAQ Table 3 05.16.2021.pdf available at https://authorea.com/ users/414500/articles/522530-measuring-transition-readiness-in-adolescents-and-youngadults-with-sickle-cell-disease-using-the-transition-readiness-assessment-questionnaire

\section{Hosted file}

MEASURING TRANSITION READINESS TRAQ Table 4 05.16.2021.pdf available at https://authorea.com/ users/414500/articles/522530-measuring-transition-readiness-in-adolescents-and-youngadults-with-sickle-cell-disease-using-the-transition-readiness-assessment-questionnaire 

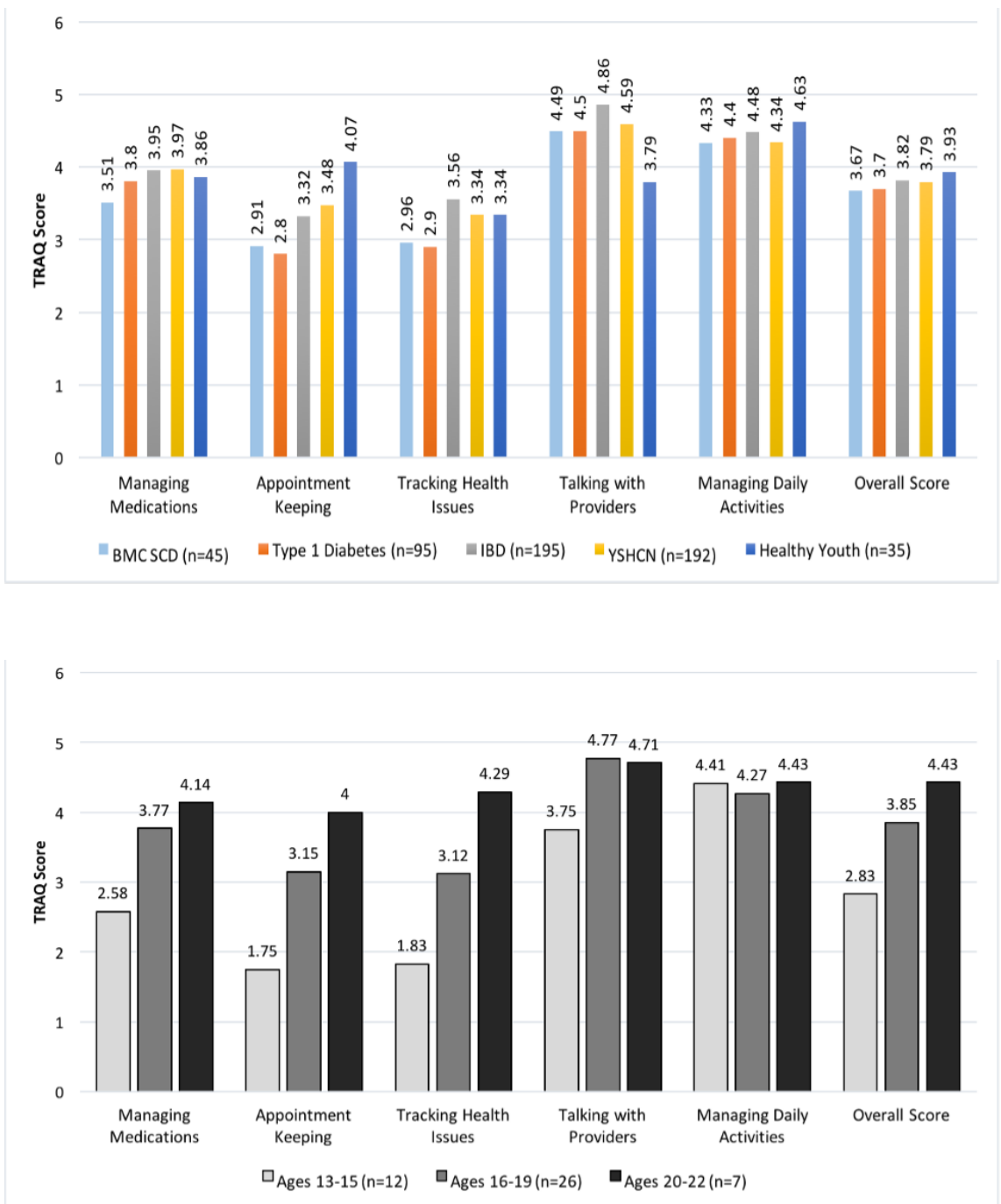